\title{
A COMMON FIXED-POINT THEOREM IN REFLEXIVE LOCALLY UNIFORMLY CONVEX BANACH SPACES ${ }^{1}$
}

\author{
MICHAEL EDELSTEIN AND MO TAK KIANG
}

\begin{abstract}
Let $X$ be a reflexive locally uniformly convex Banach space and $G$ an ultimately nonexpansive commutative semigroup of continuous self-maps of $X$. If there exists a point $x$ in $X$ recurrent under $G$ such that $G(x)$ is bounded, then $G$ has a common fixed point in $\overline{c o}(G(x))$. If $X$ is a Hilbert space then there is exactly one such point in $\overline{\operatorname{co}}(G(x))$.
\end{abstract}

1. Introduction. Let $(X, d)$ be a metric space and $G$ a family of mappings $g$ : $X \rightarrow X$ forming a semigroup under composition. The notion of a $G$-closure point $x$ was introduced in [5] and defined by the condition: for some $z \in X$, any $\varepsilon>0$, and any $f \in G$ there is a $g \in G$ such that

$$
d(f g(z), x)<\varepsilon .
$$

In [4] we discussed fixed point properties of semigroups, termed ultimately nonexpansive and defined by the condition that for every $u, v \in X$ and every $\alpha>0$ there is an $f \in G$ such that, for all $g \in G$,

$$
d(f g(u), f g(v)) \leqslant(1+\alpha) d(u, v) .
$$

Among other things it was shown there that if $X$ is a reflexive locally uniformly convex Banach space and $G$ is an ultimately nonexpansive commutative semigroup of continuous mappings $g: X \rightarrow X$, then the existence of a point $x$ with a precompact orbit $G(x)=\{g(x): g \in G\}$ guarantees a common fixed point.

It is the purpose of this paper to prove the stronger result, obtained by replacing the hypothesis of precompactness by the assumption that there exist a $G$-closure point whose orbit is bounded. The special case where $G$ is generated by a single map $f$ was treated in [3], where it was shown that the generator $f$ has a unique fixed point in $\overline{\operatorname{co}}\left\{f^{n}(x): n=1,2, \ldots\right\}$. The case of a general semigroup $G$, which is the object of this paper, is of added interest, as the $G$-closure property is, in general, weaker than the corresponding one for a single map $f$. This fact is amply reflected in the more elaborate arguments of Lemmas 1-5, which pave the way to the proof that the restriction of $G$ to $\overline{\operatorname{co}}(G(x))$ is an affine isometry (cf. §2). There seems to be no

\footnotetext{
Received by the editors June 22, 1984.

1980 Mathematics Subject Classification. Primary 47H10.

Kev words and phrases. Ultimately nonexpansive semigroup, recurrence, common fixed point, isometry, affine isometry.

${ }^{1}$ Research supported by NSERC grants A-3999 and A-8756.
} 
compelling reason to believe that the uniqueness part of [3] is valid in general, although it does hold in the case where $X$ is a Hilbert space.

To simplify the presentation of our main result, we introduce the notion of $G$-recurrence. Thus, a point $x \in X$ is said to be $G$-recurrent, or recurrent under $G$, if for any $\varepsilon>0$ and any $f \in G$ there is an $h \in G$ such that

$$
d(f h(x), x)<\varepsilon .
$$

In [4, Proposition 1(a)] we pointed out that if $G$ is an ultimately nonexpansive commutative semigroup on any metric space, then $x$ is $G$-recurrent if it is a $G$-closure point. Clearly then, for semigroups such as those in this paper, the two notions are equivalent.

\section{Preliminaries.}

LEMMA 1. Let $G$ be an ultimately nonexpansive commutative semigroup of continuous mappings of a Banach space $X$ into itself. Let $z, u_{1}, u_{2}, \ldots, u_{n}$ be members of $X$. Then to any positive integer $k$ there is $a g_{k}$ in $G$ with the property that, for any $g$ in $G$ and each $i=1,2, \ldots, n$,

$$
\left\|g g_{k}\left(u_{i}\right)-g g_{k}(z)\right\| \leqslant(1+1 / k)\left\|u_{i}-z\right\| .
$$

Proof. For a fixed $i$ there exists a $g_{k}^{(i)}$ in $G$ such that (1) is satisfied, with $g_{k}^{(i)}$ replacing $g_{k}$. Clearly then, (1) holds with $g_{k}=g_{k}^{(1)} g_{k}^{(2)} \cdots g_{k}^{(n)}$.

LeMma 2. Let $z_{1}, z_{2} \in X, g \in G$, where $G$ is as in Lemma 1 , and suppose that a sequence $\left\{g_{k}\right\} \subset G$ exists such that, for all $h \in G$,

$$
\left\|h g_{k} g\left(z_{1}\right)-h g_{k} g\left(z_{2}\right)\right\| \leqslant(1+1 / k)\left\|z_{1}-z_{2}\right\|
$$

and

$$
\left\|h g_{k} g\left(z_{1}\right)-h g_{k} g\left(z_{2}\right)\right\| \leqslant(1+1 / k)\left\|g\left(z_{1}\right)-g\left(z_{2}\right)\right\|
$$

for $k=1,2 \ldots$

Suppose further that sequences $\left\{h_{k}\right\},\left\{h_{k}^{\prime}\right\} \subset G$ exist such that $\lim _{k \rightarrow \infty} h_{k}^{\prime} g_{k} g\left(z_{i}\right)$ $=g\left(z_{i}\right)$ and $\lim _{k \rightarrow \infty} h_{k} g_{k} g\left(z_{i}\right)=z_{i}(i=1,2)$. Then

$$
\left\|g\left(z_{1}\right)-g\left(z_{2}\right)\right\|=\left\|z_{1}-z_{2}\right\| \text {. }
$$

Proof. Substituting $h_{k}^{\prime}$ and $h_{k}$ for $h$ in (2) and (3), respectively, we obtain two inequalities from which the result follows. Indeed,

$$
\left\|g\left(z_{1}\right)-g\left(z_{2}\right)\right\|=\lim _{k \rightarrow \infty}\left\|h_{k}^{\prime} g_{k} g\left(z_{1}\right)-h_{k}^{\prime} g_{k} g\left(z_{2}\right)\right\| \leqslant\left(1+\frac{1}{k}\right)\left\|z_{1}-z_{2}\right\|
$$

and

$$
\left\|z_{1}-z_{2}\right\|=\lim _{k \rightarrow \infty}\left\|h_{k} g_{k} g\left(z_{1}\right)-h_{k} g_{k} g\left(z_{2}\right)\right\| \leqslant\left(1+\frac{1}{k}\right)\left\|g\left(z_{1}\right)-g\left(z_{2}\right)\right\|,
$$

whence, since $k=1,2, \ldots$ is arbitrary, $\left\|g\left(z_{1}\right)-g\left(z_{2}\right)\right\| \leqslant\left\|z_{1}-z_{2}\right\|$ and, simultaneously, $\left\|z_{1}-z_{1}\right\| \leqslant\left\|g\left(z_{1}\right)-g\left(z_{2}\right)\right\|$. 
LEMMA 3. Let $X$ be a reflexive locally uniformly convex Banach space, and let $G$ be as in Lemma 1. Suppose that $p, q, z \in X$ and $\left\{g_{k}\right\} \subset G$ are such that

$$
z=\lambda p+(1-\lambda) q
$$

for some $\lambda, 0<\lambda<1$, and

$$
\begin{gathered}
\left\|g g_{k}(p)-g g_{k}(z)\right\| \leqslant(1+1 / k)\|p-z\|, \\
\left\|g g_{k}(q)-g g_{k}(z)\right\| \leqslant(1+1 / k)\|q-z\|
\end{gathered}
$$

for all $g \in G$.

Suppose further that a sequence $\left\{h_{k}\right\} \subset G$ exists such that $\left\{h_{k} g_{k}(p)\right\}$ and $\left\{h_{k} g_{k}(q)\right\}$ both converge and $\lim _{k \rightarrow \infty} h_{k} g_{k}(p)=p, \lim _{k \rightarrow \infty} h_{k} g_{k}(q)=q$. Then $\left\{h_{k} g_{k}(z)\right\}$ converges, and $\lim _{k \rightarrow \infty} h_{k} g_{k}(z)=z$.

Proof. In (5) we may replace $g$ by members of the sequence $\left\{h_{k}\right\}$ and observe that, since the sequences $\left\{h_{k} g_{k}(p)\right\}$ and $\left\{h_{k} g_{k}(q)\right\}$ are bounded, so is $\left\{h_{k} g_{k}(z)\right\}$. By reflexivity of $X$ some subsequence $\left\{h_{k_{j}} g_{k_{j}}(z)\right\}$ converges weakly to, say, $w \in X$. Since norms are weakly lower semicontinuous, it follows from the above inequalities that $\|p-w\| \leqslant\|p-z\|$ and $\|q-w\| \leqslant\|q-z\|$. Hence,

$$
\|p-q\|=\|p-z\|+\|q-z\| \geqslant\|p-w\|+\|q-w\| \geqslant\|p-q\|,
$$

clearly implying that $\|p-w\|=\|p-z\|$ and $\|q-w\|=\|q-z\|$. Hence, $w=z$ by strict convexity of $X$; and because this is true for each weakly convergent subsequence of $\left\{h_{k} g_{k}(z)\right\}$, it is also true that the entire sequence converges weakly to $z$.

Now the vectors

$$
[(1-1 / k)\|p-z\|]^{-1}\left(h_{k} g_{k}(p)-h_{k} g_{k}(z)\right) \quad(k=1,2, \ldots)
$$

are all of norm $\leqslant 1$ and form a sequence which converges weakly to

$$
(p-z)[\|p-z\|]^{-1}
$$

on the unit sphere. By a known property of locally uniformly convex Banach spaces (cf. [1, p. 32]), the same sequence converges in norm. Hence,

$$
\lim _{k \rightarrow \infty}\left(h_{k} g_{k}(p)-h_{k} g_{k}(z)\right)=p-z \text { and } \lim _{k \rightarrow \infty} h_{k} g_{k}(z)=z,
$$

as claimed.

LeMmA 4. Let $X$ and $G$ be as in Lemma 2. Suppose $x \in X$ is recurrent under $G$, and let $u_{1}, u_{2} \in G(x)$. Then the restriction of each member $g$ of $G$ to the line segment $\left[u_{1}, u_{2}\right]$ is an affine isometry.

Proof. Let $z_{1}, z_{2}$ be points on the line segment $\left[u_{1}, u_{2}\right]$. Since all isometries in a strictly convex Banach space are affine, it suffices to show that $\left\|g\left(z_{1}\right)-g\left(z_{2}\right)\right\|=$ $\left\|z_{1}-z_{2}\right\|$. To this end let $\left\{g_{k}\right\} \subset G$ be a sequence with the property that

$$
\left\|h g_{k} g(u)-h g_{k} g(v)\right\| \leqslant(1+1 / k)\|u-v\|
$$

and

$$
\left\|h g_{k} g(u)-h g_{k} g(v)\right\| \leqslant(1+1 / k)\|g(u)-g(v)\|
$$


for all $h \in G$, all $k=1,2, \ldots$, and all $u, v$ in the set $\left\{u_{1}, u_{2}, z_{1}, z_{2}, g\left(u_{1}\right), g\left(u_{2}\right)\right.$, $\left.g\left(z_{1}\right), g\left(z_{2}\right)\right\}$. Let $\left\{h_{k}\right\}$ be a sequence in $G$ with the property that $\lim _{k \rightarrow \infty} h_{k} g_{k} g(x)$ $=x$. Then, by the continuity of members of $G, \lim _{k \rightarrow \infty} h_{k} g_{k} g\left(u_{i}\right)=u_{i}, i=1,2$. By Lemma $3, \lim _{k \rightarrow \infty} h_{k} g_{k} g\left(z_{i}\right)=z_{i}, i=1,2$. Next, set $h_{k}^{\prime}=h_{k} g$. Then

$$
\lim _{k \rightarrow \infty} h_{k}^{\prime} g_{k} g\left(u_{i}\right)=g\left(u_{i}\right) \text { and } \lim h_{k}^{\prime} g_{k} g\left(z_{i}\right)=g\left(z_{i}\right)
$$

for $i=1,2$. Lemma 2 applies to the effect that $\left\|g\left(z_{1}\right)-g\left(z_{2}\right)\right\|=\left\|z_{1}-z_{2}\right\|$.

LEMMA 5. Let $X$ be a reflexive locally uniformly convex Banach space and $G$ an ultimately nonexpansive commutative semigroup of continuous mappings of $X$ into itself. If $x \in X$ is recurrent under $G$, then the restriction of each $g \in G$ to $\overline{\operatorname{co}}(G(x))$ is an affine isometry.

Proof. Let $n \geqslant 2$ be a positive integer and suppose that $z_{1}, z_{2} \in \operatorname{co}\left\{u_{1}, u_{2}, \ldots, u_{n}\right\}$, $z_{1} \neq z_{2}$. For $n=2,\left\|g\left(z_{1}\right)-g\left(z_{2}\right)\right\|=\left\|z_{1}-z_{2}\right\|$ by Lemma 4. Suppose this is true for $z_{1}, z_{2} \in \operatorname{co}\left\{u_{1}, u_{2}, \ldots, u_{m}\right\}$ with $m \leqslant n-1$. Let $p_{1}, p_{2}$ be extreme points of the line segment $l \cap \operatorname{co}\left\{u_{1}, u_{2}, \ldots, u_{n}\right\}$, where $l$ is the straight line through $z_{1}$ and $z_{2}$. Choose $\left\{g_{k}\right\} \subset G$ so as to satisfy the two inequalities of Lemma 2. Further, let $\left\{h_{k}\right\}$ and $\left\{h_{k}^{\prime}\right\}$ be as in the proof of Lemma 4; that is, $\lim _{k \rightarrow \infty} h_{k} g_{k} g(x)=x$ and $\lim h_{k}^{\prime} g_{k} g(x)=g(x)$. Now $p_{1}, p_{2}$ are each convex combinations of $m$ of the points of $\left\{u_{1}, u_{2}, \ldots, u_{n}\right\}$, with $m \leqslant n-1$, and $g$ is affine on the convex hull of such sets. Suppose $p_{1}=\sum_{i=1}^{m} \lambda_{i}^{\prime} u_{i}$ and $p_{2}=\sum_{i=1}^{m} \lambda_{i}^{\prime \prime} u_{i}$ for suitable $\lambda_{i}^{\prime}, \lambda_{i}^{\prime \prime}$, with $0 \leqslant \lambda_{i}^{\prime}, \lambda_{i}^{\prime \prime} \leqslant 1$ and $\sum_{i=1}^{m} \lambda_{i}^{\prime}=1=\sum_{i=1}^{m} \lambda_{i}^{\prime \prime}$. We then obtain

$$
g\left(p_{1}\right)=\sum_{i=1}^{m} \lambda_{i}^{\prime} g\left(u_{i}\right) \text { and } g\left(p_{2}\right)=\sum_{i=1}^{m} \lambda_{i}^{\prime \prime} g\left(u_{i}\right) \text {. }
$$

Hence,

$$
\lim _{k \rightarrow \infty} h_{k} g_{k} g\left(p_{1}\right)=\sum_{i=1}^{m} \lim _{k \rightarrow \infty} \lambda_{i}^{\prime} h_{k} g_{k} g\left(u_{i}\right)=\sum_{i=1}^{m} \lambda_{i}^{\prime} u_{i}=p_{1},
$$

and, similarly, $\lim h_{k}^{\prime} g_{k} g\left(p_{1}\right)=g\left(p_{1}\right)$; likewise,

$$
\lim _{k \rightarrow \infty} h_{k} g_{k} g\left(p_{1}\right)=p_{2} \text { and } \lim _{k \rightarrow \infty} h_{k}^{\prime} g_{k} g\left(p_{2}\right)=g\left(p_{2}\right)
$$

By Lemma 3 the above equations remain valid with $z_{1}, z_{2}$ replacing $p_{1}, p_{2}$. By Lemma $2,\left\|g\left(z_{1}\right)-g\left(z_{2}\right)\right\|=\left\|z_{1}-z_{2}\right\|$, and, again by strict convexity of $X, g$ is affine. Hence, $g$ is affine on $\operatorname{co}(G(x))$ and, by continuity, $g$ is also affine on $\overline{\operatorname{co}}(G(x))$.

THEOREM. Let $X$ be a reflexive locally uniformly convex Banach space and $G$ an ultimately nonexpansive commutative semigroup of continuous self-maps of $X$. If an $x \in X$ exists such that $G(x)$ is bounded and $x$ is a recurrent point under $G$, then $\overline{\operatorname{co}} G(x)$ contains a point $\xi$ such that $G(\xi)=\{\xi\}$. If, in addition, $X$ is a Hilbert space, then $\xi$ is unique with the above property; i.e., if $\eta \neq \xi$ belongs to $\overline{\operatorname{co}} G(x)$ then $g(\eta) \neq \eta$ for some $g \in G$. 
Proof. By Lemma $5, G \mid \overline{c o} G(x)$, the semigroup consisting of restrictions of members of $G$ to $\overline{c o} G(x)$, is composed of affine isometries. By the Markov-Kakutani Theorem [2] there exists a common fixed point. To prove the assertion about uniqueness, assume $\eta \neq \xi$ is another common fixed point in $\overline{\operatorname{co}} G(x)$ and let $l$ be the straight line joining $\xi$ and $\eta$. Let $\bar{g}$ be the affine isometry on the affine hull of $l \cap$ $\{\overline{\operatorname{co}} G(x)\}$, which is determined by $g \in G$. Then, for every $\alpha \in l,\|\bar{g}(x)-\bar{g}(\alpha)\|=$ $\|x-\alpha\|$. In particular, $\|\bar{g}(x)-\bar{g}(\zeta)\|=\|x-\zeta\|$, where $\zeta$ is the point of $l$ nearest to $x$. Because $x-\zeta$ is perpendicular to $l$ in an inner product space, we have $\langle x-\zeta$, $\xi-\eta\rangle=0$, and because all distances are preserved under $\bar{g}$ and $l$ is pointwise fixed, $\zeta$ is also the nearest point of $l$ to $\bar{g}(x)=g(x)$. It follows that $\langle g(x)-\zeta, \xi-\eta\rangle=0$ for all $g \in G$ and, as an easy consequence, $l \cap\{\overline{\operatorname{co}}(G(x))\}$ is a singleton. Thus, $\overline{c o} G(x)$ cannot contain $\{\xi, \eta\}$, and the proof is complete.

\section{REFERENCES}

1. J. Diestel, Geometry of Banach spaces-selected topics, Springer-Verlag, 1975.

2. N. Dunford and J. T. Schwartz, Linear operators, Part I, Interscience, New York, 1967.

3. M. Edelstein, $A$ fixed point property of reflexive locally uniformly convex Banach spaces, Math. Rep. Acad. Sci. Roy. Soc. Canada 6 (1984), 189-192.

4. M. Edelstein and M. T. Kiang, On ultimately nonexpansive semigroups, Pacific J. Math. 100 (1982), $100-110$.

5. R. D. Holmes and P. P. Narayanaswami, On asymptotically nonexpansive semigroups of mappings, Canad. Math. Bull. 13 (1970), 209-214.

Department of Mathematics, Dalhousie University, Halifax, Nova Scotia B3H 4H8, Canada

Department of Mathematics, Saint Mary's University, Halifax, Nova Scotia B3H 3C3, CANADA (Current address of M. T. Kiang)

Current address (Michael Edelstein): Department of Mathematics, Mount Allison University, Sackville, N.B., E0A 3C0, Canada 